\title{
Fechamiento arqueomagnético de flujos de lava del Holoceno provenientes del volcán Ceboruco, occidente de México
}

Gerardo Gifuentes-Nava, Miguel Ángel Cervantes-Solano, Ulises Díaz-Ortega, Avto Goguitchaichvili, Hector López-Loera, José Rosas-Elguera, Hugo Delgado-Granados, Juan Morales-Contreras, Rubén Cejudo-Ruíz, Jaime Urrutia-Fucugauchi

Gerardo Cifuentes-Nava

gercifue@geofisica.unam.m.

Programa de Posgrado en Ciencias de la Tierra, Universidad Nacional Autónoma de México, Circuito de la investigación Científica s/n, Ciudad Universitaria, Coyoacán 04510, CDMX, México.

Miguel Ángel Cervantes-Solano

Escuela Nacional de Estudios Superiores Unidad Morelia, Universidad Nacional Autónoma de México, Antigua Carretera a Pátzcuaro No. 8701, Col. Ex Hacienda de San José de la Huerta, 58190 Morelia, Michoacán, México.

Ulises Díaz Ortega

Avto Goguitchaichvili

Juan Morales Contreras

Servicio Arqueomagnético Nacional y Laboratorio Interinstitucional de Magnetismo Natural, Unidad Michoacán del Instituto de Geofisica, Universidad Nacional Autónoma de México, Antigua Carretera a Pátzcuaro No. 8701, Col. Ex Hacienda de San José de la Huerta, 58190 Morelia, Michoacán, México.

Héctor López-Loera

División de Geociencias Aplicadas, IPICYT, Camino a la Presa San José No. 2055, Col. Lomas, sección 4, 7821 San Luis Potosí, San Luis Potosí, México.

\section{José Rosas-Elguera}

Laboratorio Interinstitucional de Magnetismo Natural, Sede Universidad de Guadalajara, Av. Juárez No. 976, Edificio de la Rectoría General, piso 11, Colonia Centro, 44100 Guadaajara, Jalisco, México.

\section{Hugo Delgado-Granados}

Departamento de Vulcanología, Instituto de Geofisica, Universidad Nacional Autónoma de México, Circuito de la investigación Científica s/n, Ciudad Universitaria, Coyoacán 04510, CDMX, México.

\section{Rubén Cejudo-Ruiz}

Laboratorio Interinstitucional de Magnetismo Natural, Unidad Michoacán del Instituto de Geofísica, Universidad Nacional Autónoma de México, Antigua Carretera a Pátzcuaro No. 8701, Col. Ex Hacienda de San José de la Huerta, 58190 Morelia, Michoacán, México.

\section{Jaime Urrutia-Fucugauchi}

Laboratorio de Paleomaonetismo, Instituto de Geofisica, Universidad Nacional Autónoma de México, Circuito de la investigación Científica s/n, Ciudad Universitaria, Coyoacán 04510, CDMX, México.

BOL. SOC. GEOL. MEX. 2019

VOL. 71 NO. 2

P. $445-455$

http://dx.doi.org/10.18268/BSGM2019v7 ln2a13

\section{RESUMEN}

En el presente trabajo se aplicó el método de fechamiento arqueomagnético a rocas provenientes de cinco flujos de lava que pertenecen al campo volcánico Ceboruco en Jalisco, en el occidente de México, utilizando el modelo de predicción de campo geomagnético SHADIF.14K a partir de las inclinaciones, declinaciones e intensidades absolutas. Se estudiaron cinco flujos de lava de cinco eventos eruptivos, los cuales tienen edad establecida por métodos radiométricos de $\mathrm{C}^{14}$. En dos sitios se obtuvo un fechamiento similar al obtenido mediante el método radiométrico; un sitio presentó discrepancias con la datación radiométrica, dado que se trata de eventos volcánicos distintos (explosivo $v s$. efusivo) y las rocas usadas en este estudio fueron emplazadas en diferente tiempo. En dos sitios el fechamiento no se pudo hacer debido a que las rocas pudieron haber sufrido alguna rotación o desplazamiento posterior al emplazamiento no advertido durante el muestreo.

Palabras clave: Datación, Arqueomagnetismo, Volcán Ceboruco, Holoceno.

\section{ABSTRACT}

In this paper we present the magnetic dating results of five independent lava flows belonging to the Ceboruco volcanic field in Falisco, western Mexico, using the SHADIF14K geomagnetic field prediction model. Five lava flows corresponding to well-documented eruptive events were studied. The ages of these flows have already been established by radiometric methods $\left(C^{14}\right)$. In two sites the archaeomagnetic ages coincide with $C^{14}$ ages previously reported. In one site the archaeomagnetic age is considerably different from the radiocarbon age, possibly due to a discrepancy between one or both of the ages and the actual age of emplacement. For the two remaining lava flows, the paleomagnetic directions were found to be scattered and thus no characteristic remanence was determined. These sites probably underwent some rotation or a later displacement that was unnoticed during the sampling.

Keywords: Archaeomagnetism, Dating, Ceboruco Volcano, Holocene. 


\section{Introducción}

Un amplio campo de aplicación del arqueomagnetismo es su uso como herramienta de fechamiento de rocas ya que, bajo ciertas condiciones, éstas registran las características del campo magnético terrestre (GMT) existente en el momento de su formación. Tal información se puede obtener mediante el estudio de la magnetización remanente en la roca. Si se conocen con precisión las variaciones del CMT en el pasado, es posible establecer un registro de variación temporal (curva de variación secular), el cual puede utilizarse como método de fechamiento; este método es conocido como fechamiento paleomagnético/arqueomagnético y puede ser tan preciso como el método de fechamiento radiométrico (Tauxe, 2010). El método por $\mathrm{C}^{14}$ se usa con mayor frecuencia para fechar rocas del Pleistoceno Tardío y Holoceno ( $<40$ ka), sin embargo, este fechamiento se hace frecuentemente con el carbón asociado a la formación rocosa o paleosuelo que se encuentra debajo o sobre y no directamente en la roca, lo que puede ocasionar una interpretación incorrecta de la edad del emplazamiento (Siebe et al., 2004); en cambio, el método arqueomagnético posee una ventaja significativa ya que permite fechar el momento de formación de las rocas. Por otro lado, los métodos radiométricos por $\mathrm{K} / \mathrm{Ar}$ y ${ }^{40} \mathrm{Ar} /{ }^{39} \mathrm{Ar}$ fechan directamente la roca de gran antigüedad, pero actualmente se han convertido ya en un método alternativo para fechar lavas formadas durante los últimos 14000 años (e.g. Pavón-Carrasco et al., 2011 ; Roperch et al., 2015).

Dentro de la Faja Volcánica Trans-Mexicana (FVTM) existen algunos campos volcánicos potencialmente aptos para utilizar el método arqueomagnético (e.g. Sieron y Siebe, 2008; Pérez-Rodríguez et al., 2015; García-Quintana et al., 2016; Mahgoub et al., 2017). Además, el estudio de aparatos volcánicos con actividad reciente permite refinar la curva de variación secular para diferentes regiones. En particular, el campo volcánico Ceboruco, en Nayarit y Jalisco $\left(21^{\circ} 7^{\prime} 30^{\prime} \mathrm{N}\right.$ $\left.104^{\circ} 30^{\prime} 30^{\prime} \mathrm{O}\right)$, representa una excelente oportuni- dad para poder aplicar el método de fechamiento arqueomagnético, ya que presenta vulcanismo reciente y cuenta con varios fechamientos radiométricos y descripciones detalladas de los eventos eruptivos. En el presente trabajo se presentan los resultados de un estudio paleomagnético realizado a cinco flujos de lava del volcán Ceboruco en Jalisco, realizado con el objetivo de determinar las edades de los emplazamientos mediante el modelo de variación del CMT para los últimos 14000 años.

\section{Descripción del área de estudio, localización y muestreo de los flujos de lava estudiados}

Según Nelson (1980), el campo volcánico Ceboruco-San Pedro ocupa un área de 1600 km² cuenta con 70 chimeneas y 16 conos de ceniza monogenéticos, y se encuentra entre los estados de Jalisco y Nayarit en la parte occidental de la FVTM. El volcán predominante de este campo es el volcán Ceboruco, un estratovolcán Cuaternario compuesto principalmente por andesitas además dacitas, con dos calderas concéntricas anidadas, resultado de una erupción Pliniana y el colapso posterior de una cúpula interna (Frey et al., 2004). Las relaciones estratigráficas de la erupción Pliniana Jala ocurrida hace $\sim 1000$ años indican que después de ésta se han producido siete flujos de lava mayores, los cuales fueron emitidos desde el cráter central y hacia los flancos, además de múltiples conos y domos de lava ubicados en la cima en las colindancias del cráter. La erupción más reciente de este volcán ocurrió en 1870 (Caravantes, 1870; Iglesias et al., 1877). Sieron y Siebe (2008) describen detalladamente la historia eruptiva de este volcán y señalan que es difícil determinar las edades de las erupciones posteriores a la erupción con el método radiométrico debido a que los afloramientos no cuentan con suficiente material útil para el fechamiento.

La estrategia de muestreo estuvo determinada principalmente por dos condiciones: recolectar 
muestras del flujo de lava de la erupción bien documentada de 1870 y de los flujos de lava de edades determinadas mediante el método radiométrico de $\mathrm{C}^{14}$. Debido a que las muestras utilizadas para los fechamientos son fragmentos de carbón encontrados dentro de flujos piroclásticos o paleosuelos (Sieron y Siebe, 2008; Nelson, 1980), en dos casos fue necesario utilizar fotografías aéreas y mapas de elevación digital para determinar la extensión del flujo de lava asociado y entonces recolectar en ese sitio las muestras (sitios CB_01 y CB_02 en nuestro estudio, 04-24 y 04-31, respectivamente, en Sieron y Siebe, 2008), en el resto de los casos se recolectaron las muestras directamente de los flujos de lava referidos. Se recolectaron 47 núcleos para estudio paleomagnético estándar de cinco flujos de lava distintos alrededor del volcán (de 8 a 10 núcleos por flujo, Figura 1). Todas las muestras fueron orientadas con compás magnético y en algunos casos con compás solar.

Con el propósito de estimar la posible influencia de las anomalías magnéticas locales asociadas a los sitios estudiados, se elaboró un mapa aeromagnético compuesto de toda la región aledaña al volcán y que comprende a los sitios estudiados. La información se obtuvo a partir de un vuelo hecho

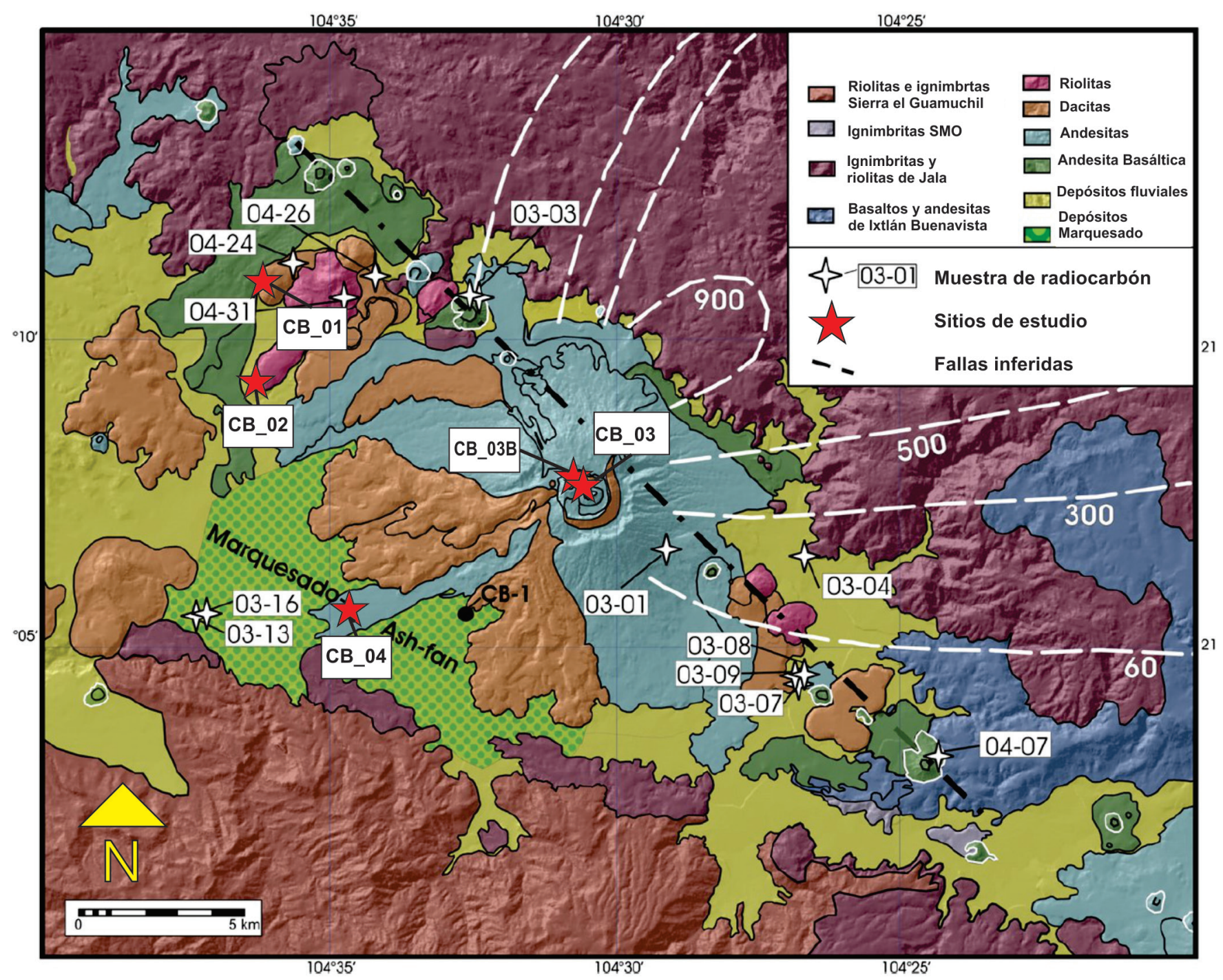

Figura 1 Detalle de los flujos de lava del volcán Ceboruco y sus alrededores (modificado de Sieron y Siebe, 2008). Los sitios de muestreo se indican con una estrella roja. 
por el Consejo de Recursos Minerales (CRM, actualmente Servicio Geológico Mexicano) en 1983, en el cual se usó un magnetómetro Geometrics G-803, con una sensibilidad de $0.25 \mathrm{nT}$, el magnetómetro de la estación base fue un Geometrics 856 A con una sensibilidad de $1 \mathrm{nT}$; se utilizó un radar altimétrico Sperry y el sistema de navegación fue visual. Los valores del campo magnético (IGRF epoch 1980.0) para la fecha del vuelo eran: Intensidad del campo 43401 nT; Inclinación 47²44’ y
Declinación 852’. Los sitios estudiados se observan en la Figura 2, que muestra el mapa con la configuración de los isovalores del campo magnético reducido al polo (Baranov y Naudy, 1964). El sitio CB_01 se encuentra en la porción NO a $9560 \mathrm{~m}$ al NO de la anomalía principal, ubicada en el centro del mapa aeromagnético. Se ubica en el gradiente $\mathrm{O}$ de una anomalía aeromagnética secundaria de forma alargada con dirección principal N-S con una longitud de $3476 \mathrm{~m}$, que en

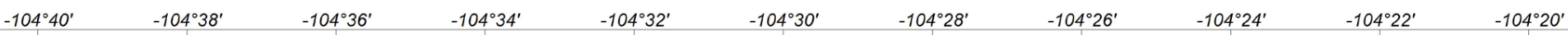

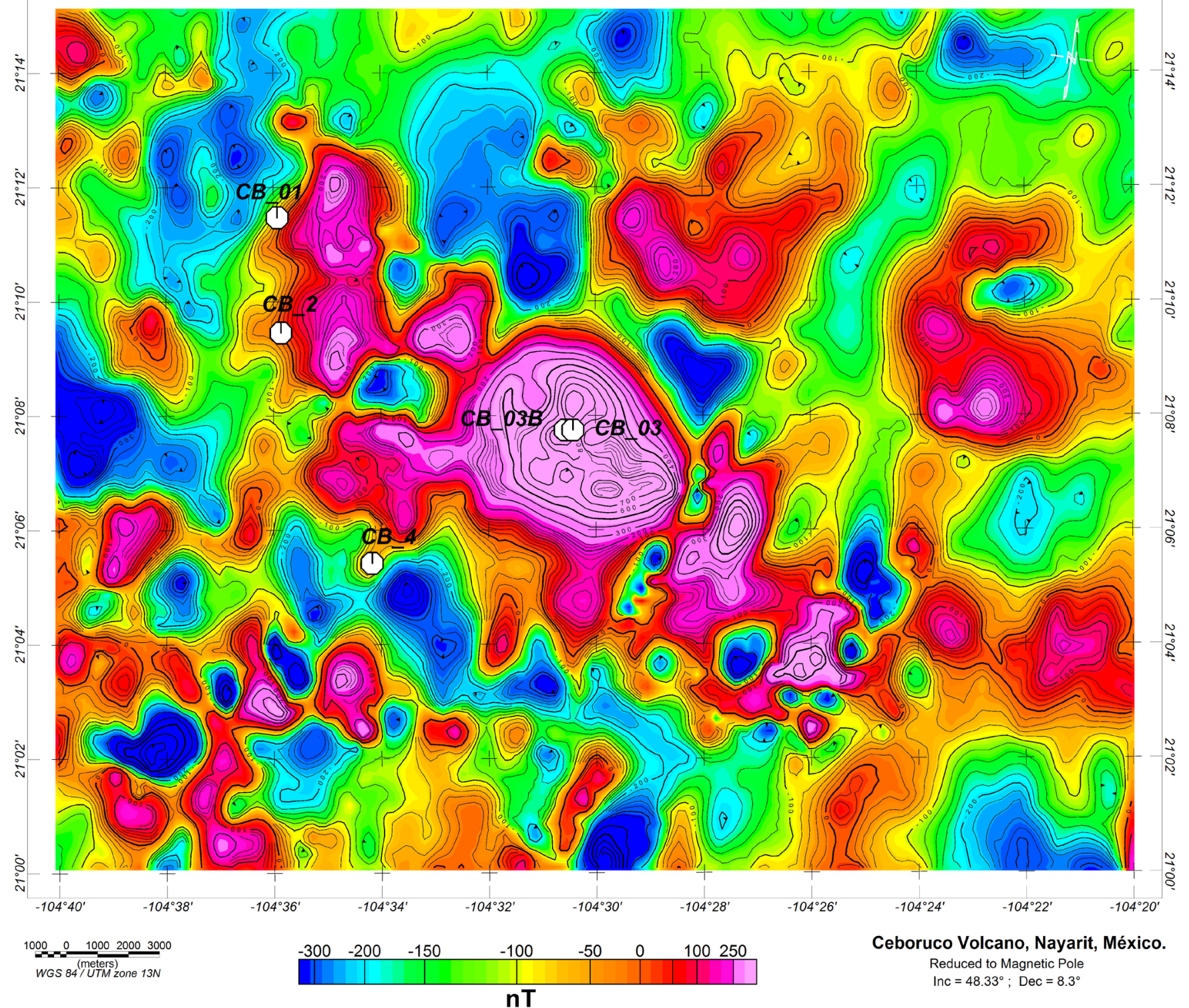

Figura 2 Mapa de la anomalía magnética reducida al polo del volcán Ceboruco en el que se muestran las principales estructuras magnéticas alrededor de los sitos de estudio. 
dirección E-O tiene $3241 \mathrm{~m}$. El sitio del muestreo está a 860 msnm y un valor de intensidad aeromagnético de $14.4 \mathrm{nT}$.

El sitio CB_02 se localiza también en la porción NO del mapa y al S de la CB_01, a una distancia de $3729 \mathrm{~m}$, a una altura de $1199 \mathrm{msnm}$ y muestra una intensidad de magnetización aeromagnética de -78 nT. La anomalía aeromagnética secundaria a la que está asociada este sitio está elongada en dirección NS, con una longitud de 4109 m, mientras que en la dirección E-O es de 3269 m. Esta anomalía concurrente con el sitio de muestreo se ubica en la porción NO del patrón aeromagnético que se observa en el mapa de reducción al polo.

Los sitios CB_03 y CB_03B se ubican en el centro del mapa aeromagnético. La configuración de isovalores muestra un patrón aeromagnético con rumbo NO-SE en el que se destaca la anomalía central que está asociada al cráter. Estos sitios están correlacionados a una gran anomalía aeromagnética de forma semicircular con dimensiones de 7960 m en dirección NO-SE y de 6825 m en dirección NE-SO del volcán Ceboruco. La muestra CB_03 se asocia a una intensidad de magnetización del orden de los $875.5 \mathrm{nT}$ y a una altitud de $2145 \mathrm{~m}$, y la CB_3B se correlaciona a una intensidad de magnetización del orden de los 856.3 m y se encuentra a una altitud de $2168 \mathrm{~m}$.

El sitio CB-04 se localiza al SO de la anomalía principal. Se asocia también a un pequeño vértice de anomalía de forma alargada en dirección NE-SO de 1795 m de longitud y 745 m de ancho. Este sitio se ubica a una altitud de $929 \mathrm{~m}$ y tiene una intensidad de magnetización del orden de los $-45 \mathrm{nT}$.

\section{Direcciones paleomagnéticas y remanencia magnética}

Con la finalidad de obtener las direcciones características de la remanencia magnética presente en las muestras, se realizaron procesos de desmagnetización mediante campos alternos en el Laboratorio Interinstitucional de Magnetismo Natural
(LIMNA) del Instituto de Geofísica de la UNAM. Se desmagnetizaron de 6 a 8 muestras por sitio aplicando campos magnéticos con intensidades desde $5 \mathrm{mT}$ hasta $90 \mathrm{mT}$ con incrementos graduales de $5 \mathrm{mT}$. Las componentes de la remanencia para cada muestra y las direcciones paleomagnéticas medias por sitio se determinaron mediante el método de análisis de componentes principales (Kirschvink, 1980) y, posteriormente, con la estadística de Fisher (1953). En la mayoría de las muestras estudiadas $(60 \%)$ se observó una sola componente estable con un comportamiento unidireccional hacia el origen de coordenadas en los diagramas ortogonales de Zijderveld et al. (1967) (Figura 3, muestra 94U007A). En otros casos, el $30 \%$ de las muestras, se observó también una segunda componente de magnetización poco significativa probablemente de origen viscoso, la cual fue fácilmente removida en las primeras etapas de desmagnetización (Figura 3, muestra 94U027A). Finalmente, en algunos casos no fue posible desmagnetizar por completo las muestras aplicando campos de hasta $90 \mathrm{mT}$, sin embargo, se observó un comportamiento lineal hacia el origen de coordenadas, por lo que se considera que es posible aislar las componentes direccionales características (Figura 3, muestras 94U031A y 94C043A).

Para determinar la naturaleza y estabilidad térmica de los portadores magnéticos presentes en las rocas estudiadas, se llevaron a cabo experimentos de susceptibilidad magnética en función de la temperatura (curvas termomagnéticas continuas K-T). Se usó un susceptibilímetro AGICO Kappa Bridge modelo MFK1, para calentar las muestras desde la temperatura ambiente hasta los $650{ }^{\circ} \mathrm{C}$ en presencia de gas argón, para reducir la posible oxidación durante el calentamiento. Las curvas termomagnéticas indican la existencia de dos fases magnéticas durante el calentamiento con temperaturas de Curie cercanas a los $320^{\circ} \mathrm{C}$ y de 560 ${ }^{\circ} \mathrm{C}$, lo cual apunta a la titanomagnetita con contenido variable en titanio como la responsable de la magnetización. Las curvas de enfriamiento son similares a las de calentamiento mostrando una ligera disminución de la susceptibilidad inicial, la 
A)
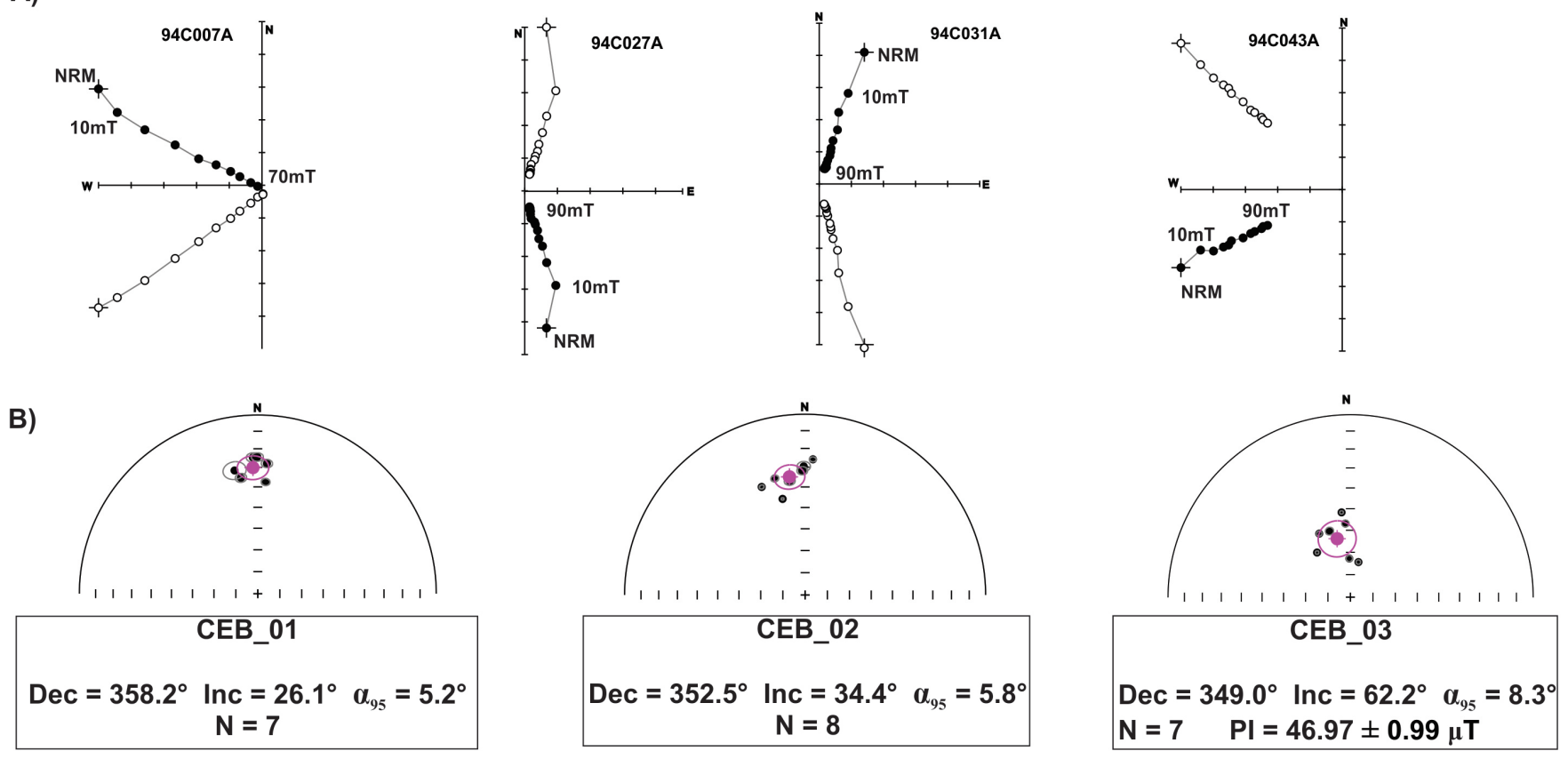

Figura 3 Diagramas ortogonales de áreas iguales con las direcciones magnéticas promedio para los sitios de estudio empleados para el fechamiento arqueomagnético.

cual es producto de la oxidación producida durante el calentamiento, Figura 4.

La determinación de intensidad paleomagnética absoluta fue hecha por el método de doble calentamiento de Thellier (Thellier y Thellier, 1959) modificado por Coe (1967). El calentamiento y enfriamiento se llevó acabo en un horno ASG TD-48 en presencia de aire. Las determinaciones de la paleointensidad se llevaron a cabo en 12 pasos de temperatura entre la temperatura ambiente y $540{ }^{\circ} \mathrm{C}$, temperatura a la cual la magnetización de la mayoría de los especímenes era removida por completo. La intensidad del campo de laboratorio aplicado fue de $45 \mu \mathrm{T}$ lográndose mantener con una precisión de hasta $0.1 \mu \mathrm{T}$ durante el experimento. Se llevaron a cabo controles de calentamiento pTRM-checks (e.g. Thellier y Thellier, 1959). Para que una determinación de paleointensidad fuera considerada confiable debió cumplir con los siguientes parámetros de calidad: (i) En el diagrama NRM-pTRM, el número de puntos alineados $\mathrm{N} \geq 5$, (ii) el factor de fracción de NRM f (Coe et al., 1978) > 0.5, (iii) el radio b (error estándar/pendiente absoluta de la línea de mejor ajuste del diagrama de Arai) $<0.1$ (Arai, 1963), (iv) el factor de calidad q $>5$ (Coe et al., 1978) y (v) los resultados de paleointensidad obtenidos de los diagramas NRM-pTRM no deben tener forma cóncava, debido a que en esos casos es probable que la remanencia esté asociada a la presencia de granos multidominio (Levi y Merri1l, 1978). Luego de realizar los experimentos, dos muestras provenientes del sitio CEB_03 superaron los criterios, obteniéndose así determinaciones exitosas para este sitio, que arrojó un valor promedio de PI $=46.97 \pm 0.99 \mu$ T, Tabla 1, Figura 5 .

\section{Fechamiento arqueomagnético}

El fechamiento arqueomagnético se obtuvo con el modelo de variación del campo magnético Terrestre propuesto por Pavón-Carrasco et al. (2014). Este modelo fue desarrollado empleando datos arqueomagnéticos y de flujos de lava distribuidos globales e incluye además los registros históricos del modelo GUFM1 (Jackson et al., 2000). El modelo SHADIF.14k permite determinar curvas de 

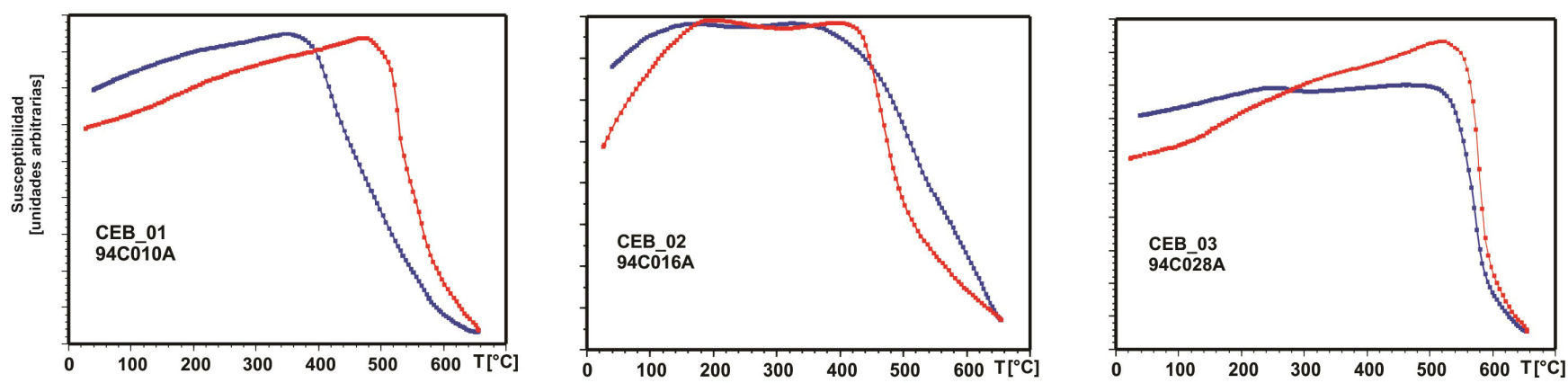

Figura 4 Curvas termomagnéticas continuas K-T. Las curvas de color rojo indican el calentamiento y las rojas el enfriamiento, la susceptibilidad magnética se muestra en unidades arbitrarias.

variación paleosecular con sus incertidumbres desde el año 12000 antes de Cristo hasta el año 1900 de nuestra era. Este modelo puede usarse como herramienta de fechamiento, ya que permite hacer una comparación de los datos direccionales y de intensidad de los sitios estudiados con los de la curva de variación correspondiente, de esta forma es posible proporcionar un intervalo de edad probable.

El fechamiento arqueomagnético pudo obtenerse en tres sitios, CB_01, CB_02 y CB_03, mientras que en dos sitios el método no pudo ser aplicado debido a la dispersión de los datos direccionales y de intensidad magnética, por lo que se obtienen varios intervalos de edades igualmente probables haciendo ambigua la determinación. La causa probable es que las muestras fueron tomadas de bloques de roca rotados o desplazados posteriormente al emplazamiento, lo cual no fue advertido durante el muestreo. En la Tabla 2 (Figura 6) se muestran los resultados del fechamiento y la edad radiométrica reportada previamente.

Para el sitio CB_01, utilizando únicamente los datos direccionales, se obtuvo como intervalo de edad más probable 735 - 933 DC. Este intervalo difiere del reportado por Sieron y Siebe (2008) de $996-1168$ DC. La diferencia puede estar asociada a que, según el mismo artículo, la estimación de la edad se basó en determinaciones individuales realizadas en muestras de carbón o bien de paleosuelos asociados a los productos de las erupciones y no directamente a los afloramientos. En cambio, el fechamiento de este estudio fue hecho utilizando muestras de roca del afloramiento, por lo que podría tratarse de dos eventos eruptivos diferentes muy cercanos en tiempo, primero la erupción, la emisión de lavas y posteriormente una etapa eruptiva explosiva, por lo cual es más probable que la edad reportada por el presente estudio corresponda al inicio de la actividad volcánica eruptiva y concuerde con el inicio de la actividad de la erupción Pliniana de Jala.

En el sitio CB_02, con datos direccionales, se obtuvo un intervalo de edad probable de 192 - 69 AC. Esta edad difiere a la reportada por Sieron y Siebe (2008) de 734 - 690 AC. En este caso la diferencia puede atribuirse a que la edad que reportan se obtuvo mediante muestras de carbón obtenidas en depósitos freatomagmáticos, mientras que nuestra determinación se obtuvo a partir de una muestra de roca proveniente del afloramiento de lava de la erupción. Nuevamente la diferencia en las edades podría indicar la temporalidad de dos eventos eruptivos cercanos en tiempo, la emisión de lavas y posteriormente la erupción explosiva, por lo que, a pesar de esta diferencia, esta nueva determinación concuerda con la historia eruptiva previa a la erupción de Jala y es probable que el flujo de lava sea anterior a la erupción reportada por Sieron y Siebe (2008).

Para el sitio CEB_03, además de los datos direccionales, se incluyó el valor de la paleointensidad promedio $(46.97 \pm 0.99 \mu \mathrm{T})$ para determinar la edad, lo cual produjo un intervalo probable de 

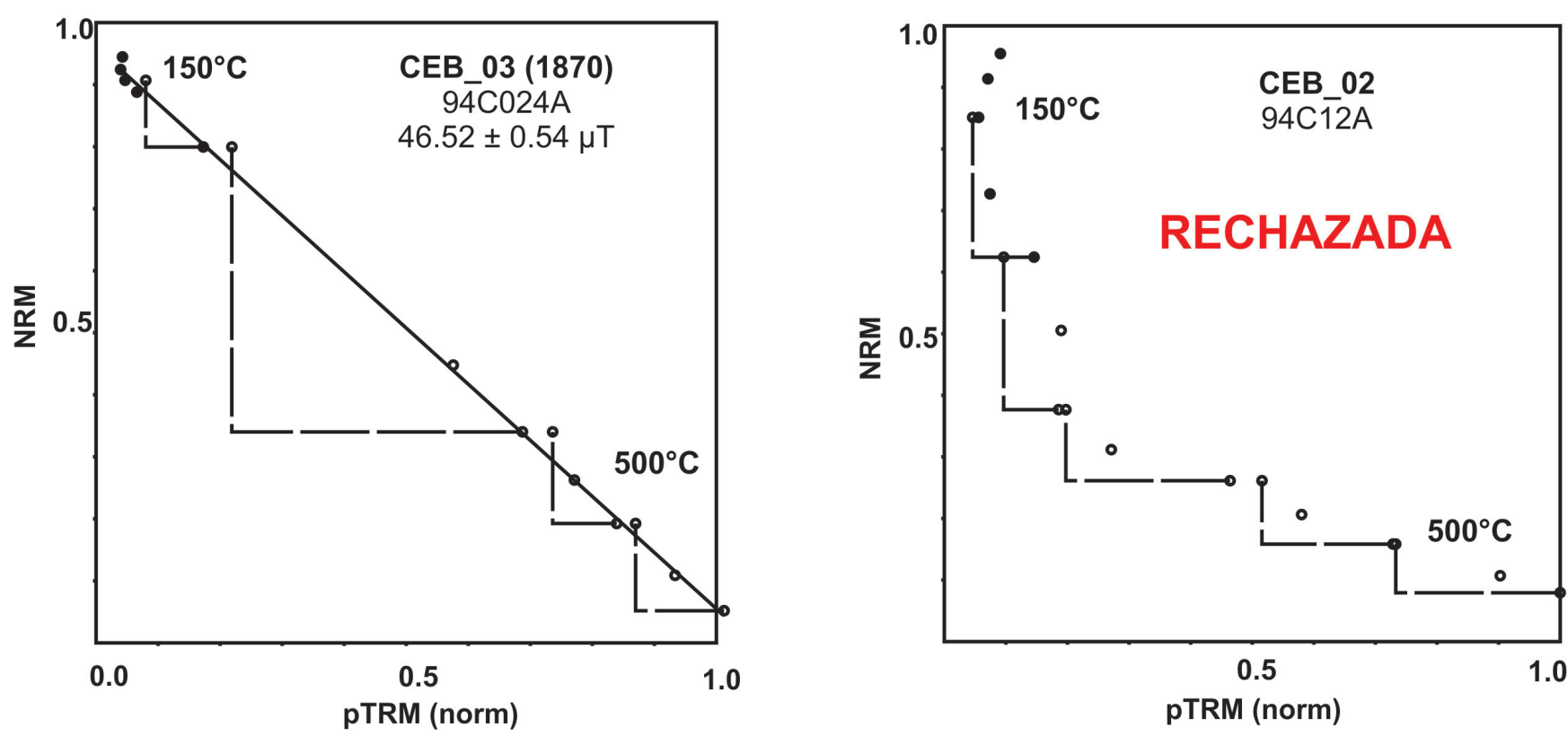

Figura 5 Diagramas de Arai-Nagata (Arai, 1963) de los experimentos de paleointensidad según el método de Thellier-Thellier (datos representativos).

Tabla 1. Resultados de paleointensidad. $\boldsymbol{n}$ es el número de pasos de calentamiento usados para la determinación de la paleointensidad, Tmin-Tmax es el intervalo de temperatura empleado para la determinación, $F \pm \Delta F$ es el valor de la paleointensidad en micro Teslas y su correspondiente desviación estándar, $f$ es la fracción de NRM utilizada para la determinación, $g$ y $q$ son los factores de calidad definidos por Coe et al. (1978).

\begin{tabular}{|c|c|c|c|c|c|c|c|}
\hline Sitio & Muestra & $\mathbf{N}$ & $\begin{array}{c}\mathbf{T}_{\max }-\mathbf{T}_{\min } \\
{ }^{\circ} \mathbf{C}\end{array}$ & $\boldsymbol{f}$ & $\boldsymbol{g}$ & $\boldsymbol{q}$ & $\begin{array}{c}\boldsymbol{F} \pm \Delta \boldsymbol{F} \\
\boldsymbol{\mu T}\end{array}$ \\
\hline & 94C023A & 11 & $540-200$ & 0.94 & 0.78 & 76.73 & $47.42 \pm 0.45$ \\
\hline CB_03 & 94C024A & 11 & $540-200$ & 0.97 & 0.77 & 64.23 & $46.52 \pm 0.54$ \\
& & & & & & PROM. & $\mathbf{4 6 . 9 7} \pm \mathbf{0 . 9 9}$ \\
\hline
\end{tabular}

1810 - 1900 DC. Este sitio corresponde a una erupción histórica bastante bien documentada del año de 1870 (García, 1875; Iglesias et al., 1877), la cual queda incluida dentro del intervalo. Este mismo flujo fue estudiado por Böhnel et al. (2016), quienes, empleando la misma herramienta de fechamiento y un número mayor de muestras, reportaron resultados similares a los nuestros (1755 - 1871 DG). La coincidencia de ambas determinaciones con la edad real de la erupción pone de manifiesto la confiabilidad del método arqueomagnético como herramienta de fechamiento.

\section{Conclusiones}

La aplicación del método de fechamiento arqueomagnético mediante el modelo de variación del campo magnético SHA.DIF.14K a rocas provenientes de cinco flujos de lava que pertenecen al campo volcánico Ceboruco en Jalisco, permite establecer con mayor certeza edades de los emplazamientos. En el sitio CB_01 la nueva edad encontrada concuerda con el inicio del periodo eruptivo previamente reportado conocido como la erupción Pliniana Jala. En el sitio CB_02 la de- 

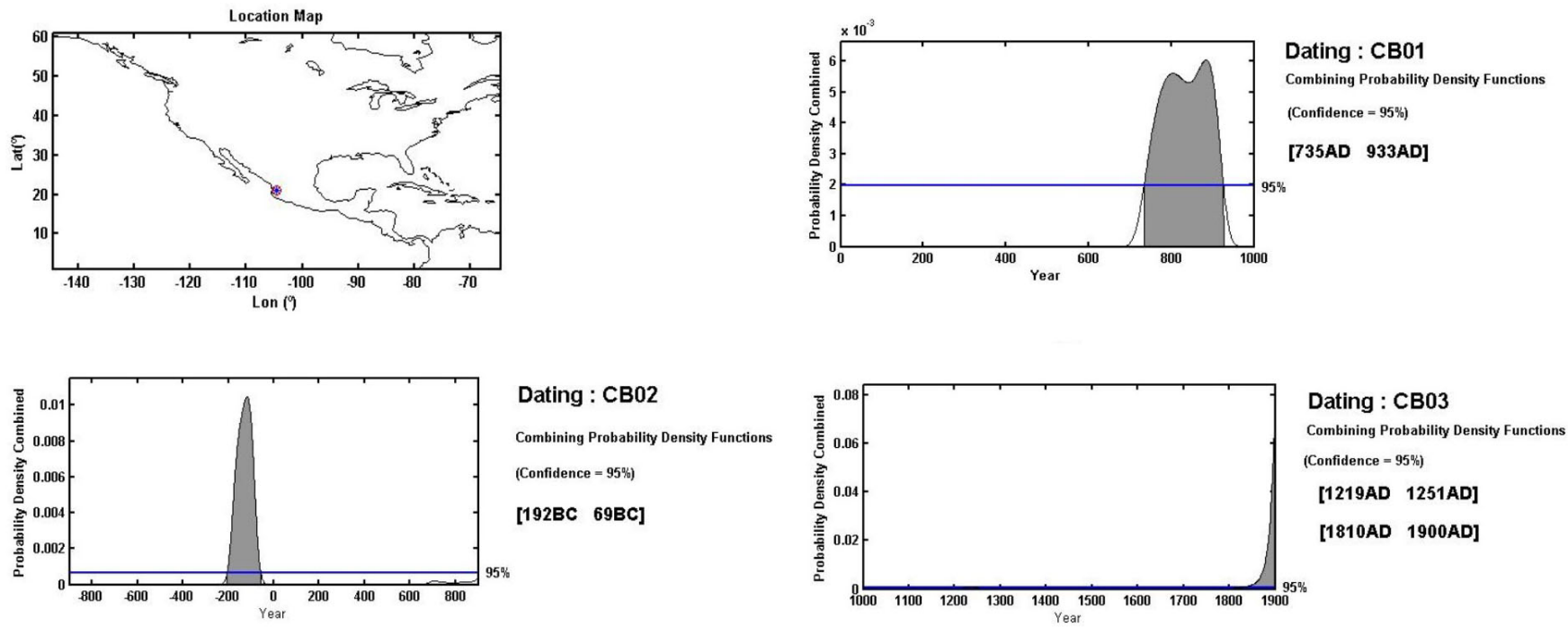

Figura 6 Fechamiento de los flujos de lava. Se muestran las gráficas de probabilidad combinada al 95\% de confianza; dentro de los corchetes se indica el intervalo de edad más probable. Estas gráficas fueron hechas con la herramienta de Matlab ARCHAEODATING de Pavón Carrasco et al. (2011).

Tabla 2. Resumen de los datos paleomagnéticos y las edades obtenidas. Lat. y Long. son la latitud y longitud geográfica de los sitios de estudio, Dec. e Inc. son la declinación e inclinación magnética respectivamente, $a_{95}$ es el radio del cono del $95 \%$ de confianza de la estadística de Fisher.

\begin{tabular}{|c|c|c|c|c|c|c|c|c|c|}
\hline Flujo & $\begin{array}{l}\text { Lat. } \\
\left({ }^{\circ} \mathrm{N}\right)\end{array}$ & $\begin{array}{l}\text { Long. } \\
\left({ }^{\circ} \mathrm{O}\right)\end{array}$ & $\begin{array}{l}\text { Dec. } \\
\left({ }^{\circ}\right)\end{array}$ & $\begin{array}{l}\text { Inc. } \\
\left({ }^{\circ}\right)\end{array}$ & $\begin{array}{l}\alpha_{95} \\
\left({ }^{\circ}\right)\end{array}$ & $\mathbf{k}$ & $\begin{array}{l}\text { Intervalo de edad } \\
\text { más probable }\end{array}$ & $\begin{array}{c}\text { Edad previamente } \\
\text { reportada }\end{array}$ & Fuente consultada \\
\hline CB_01 & $21^{\circ} 09.479^{\prime}$ & $104^{\circ} 35.871^{\prime}$ & 358.2 & 26.1 & 5.2 & 115 & 735 - 933 DC & $996-1168 \mathrm{DC}$ & $\begin{array}{c}\text { Sieron y Siebe, } \\
2008\end{array}$ \\
\hline CB_02 & $21^{\circ} 11.494^{\prime}$ & $104^{\circ} 35.932^{\prime}$ & 352.5 & 34.4 & 5.8 & 83 & $192-69 \mathrm{AC}$ & $734-690 \mathrm{AC}$ & $\begin{array}{c}\text { Sieron y Siebe, } \\
2008\end{array}$ \\
\hline CB_03 & $21^{\circ} 07.773^{\prime}$ & $104^{\circ} 30.442^{\prime}$ & 349 & 62.2 & 8.3 & 54 & $1810-1900 \mathrm{DC}$ & 1870 & $\begin{array}{l}\text { Documentos } \\
\text { historicos }\end{array}$ \\
\hline CB_03B & $21^{\circ} 07.779^{\prime}$ & $104^{\circ} 30.547^{\prime}$ & 18.8 & 57.2 & 6.3 & 93 & ND & --- & --- \\
\hline CB_04 & $21^{\circ} 05.464^{\prime}$ & $104^{\circ} 34.184^{\prime}$ & 249.8 & -38.4 & 6.4 & 109 & ND & --- & --- \\
\hline
\end{tabular}

terminación de la edad paleomagnética difiere de la edad radiométrica previamente reportada; esta diferencia puede atribuirse al origen de las distintas muestras usadas en ambas determinaciones, por lo que según nuestro fechamiento el flujo de lava es anterior a la erupción reportada por Sieron y Siebe (2008).

La edad encontrada para el sitio CB_03 coincide con los documentos históricos, 1870 DC, y concuerda también con una edad paleomagnética reportada recientemente. Esto nos permite considerar a esta determinación como un ejemplo de confiabilidad del método arqueomagnético como herramienta para fechar erupciones volcánicas recientes. A grandes rasgos se puede afirmar que las nuevas edades paleomagnéticas encontradas concuerdan con la historia eruptiva del volcán, aunque requiere de un mayor muestreo y trabajo de mayor detalle para definir el inicio del periodo del emplazamiento de lava. 


\section{Agradecimientos}

Este trabajo fue apoyado por el proyecto UNAM PAPIIT IA106517. AG agradece el apoyo del Proyecto CONACYT No. 251249 y el apoyo parcial de UANAM PAPIIT 101717.

\section{Referencias}

Arai, Y., 1963, Secular variation in the intensity of the past geomagnetic field:Tokyo, University of Tokyo, MSc thesis, $84 \mathrm{p}$.

Baranov, V., Naudy, H., 1964, Numerical calculation of the formula of reduction to the magnetic pole: Geophysics, 29(1), 67-79.

Böhnel, H., Pavón-Carrasco, F.J., Sieron, K., Mahgoub, A.N., 2016, Palaeomagnetic dating of two recent lava flows from Ceboruco volcano, western Mexico: Geophysical Journal International, 207(2), 1203-1215.

Caravantes, A., 1870, El Ceboruco: La Naturaleza, Periódico Científico de la Sociedad Mexicana de Historia Natural, México, Imprenta de Ignacio Escalante, Tomo 1, 248-252.

Coe, R.S., 1967, Paleo-intensities of the Earth's magnetic field determined from Tertiary and Quaternary rocks: Journal of Geophysical Research, 72(12), 3247-3262.

Coe, R.S., Grommé, S., Mankinen, E.A., 1978, Geomagnetic paleointensities from radiocarbon-dated lava flows on Hawaii and the question of the Pacific nondipole low: Journal of Geophysical Research: Solid Earth, 83, 1740-1756.

Fisher, R., 1953, Dispersion on a sphere: Proceedings of the Royal Society of London, Mathematical, Physical and Engineering Sciences, 217, 295-305.

Frey, H.M., Lange, R.A., Hall, C.M., DelgadoGranados, H., 2004, Magma eruption rates constrained by ${ }^{40} \mathrm{Ar} /{ }^{39} \mathrm{Ar}$ chronology and GIS for the Ceboruco-San Pedro volcanic field, western Mexico: Geological Society of America Bulletin, 116, 259-276.
García, S., 1875, Una visita al pueblo de S. Cristóbal. Viaje al Ceboruco: Informe y colección de artículos relativos a los fenómenos geológicos verificados en Jalisco en el presente año y en épocas anteriores: Guadalajara, Edición oficial, Tipografía de S. Banda, Tomo II, 354 p.

García-Quintana, A., Goguitchaichvili, A., Morales, J., Cervantes-Solano, M., Osorio-Ocampo, S., Macías, J.L., UrrutiaFucugauchi, J., 2016, Datación magnética de rocas volcánicas formadas durante el Holoceno: caso de flujos de lava alrededor del Lago de Pátzcuaro (campo volcánico Michoacán-Guanajuato): Revista Mexicana de Ciencias Geológicas, 33(2), 209-220.

Iglesias, M., Bárcena, M., Matute, J.I., 1877, El Ceboruco: Anales del Ministerio de Fomento de México, 1, 168-196.

Jackson, A., Jonkers, A.R., Walker, M.R., 2000, Four centuries of geomagnetic secular variation from historical records: Philosophical Transactions of the Royal Society of London, Mathematical, Physical and Engineering Sciences, 358(1768), 957-990.

Kirschvink, J.L., 1980, The least-squares line and plane and the analysis of palaeomagnetic data: Geophysical Journal of Royal Astronomical Society, 62(3), 699-718.

Levi, S., Merrill, R.T., 1978, Properties of single-domain, pseudo-single-domain, and multidomain magnetite: Journal of Geophysical Research: Solid Earth, 83, 309-323.

Mahgoub, A.N., Böhnel, H., Siebe, G., Chevrel, M.O., 2017, Paleomagnetic study of El Metate shield volcano (Michoacán, Mexico) confirms its monogenetic nature and young age ( $1250 \mathrm{CE})$ : Journal of Volcanology and Geothermal Research, 336, 209-218.

Nelson, S.A., 1980, Geology and petrology of Volcan Ceboruco, Nayarit, Mexico: Geological Society of America Bulletin, 91, 2290-2431. 
Pavón-Carrasco, FJ., Rodríguez-Gonzáles, J., Osete, M.L., Torta, J.M., 2011, A Matlab tool for archaeomagnetic dating: Journal of Archaeological Science, 32(8), 408-419.

Pavón-Carrasco, F.J., Osete, M.L., Torta, J.M., Santis, A.D., 2014, A geomagnetic field model for the Holocene based on archaeomagnetic and lava flow data: Earth and Planetary Science Letters, 388, 98-109.

Pérez-Rodríguez, N., Morales-Contreras, J., García-Tenorio, F., Goguitchaichvili, A., 2015, Fechamiento arqueomagnético de las últimas tres erupciones plinianas del volcán Popocatépetl, en Rapalini, A.E., CaballeroMiranda, C., Goguitchaichvili, A. (eds.), Proceedings São Paulo, Brasil: São Paulo, Latinmag Letters, Special Issue, 6, C1 1, 1-7.

Roperch, P., Chauvin, A., Lara, L.E., Moreno, H., 2015, Secular variation of the Earth's magnetic field and application to paleomagnetic dating of historical lava flows in Chile: Physics of the Earth and Planetary Interiors, 242, 65-78.
Siebe, C., Rodríguez-Lara, V., Schaaf, P., Abrams, M., 2004, Radiocarbon ages of Holocene Pelado, Guespalapa, and Chichinautzin scoria cones, south of Mexico City: implications for archaeology and future hazards: Bulletin of Volcanology, 66(3), 203-225.

Sieron, K., Siebe, G., 2008, Revised stratigraphy and eruption rates of Ceboruco stratovolcano and surrounding monogenetic vents (Nayarit, Mexico) from historical documents and new radiocarbon dates: Journal of Volcanology and Geothermal Research, 176(2), 241-264.

Tauxe, L., 2010, Essentials of Paleomagnetism: Berkeley, University of California Press, 489 p.

Thellier, E., Thellier, O., 1959, Sur l'intensité du champ magnétique terrestre dans le passé historique et géologique: Annales de Géophysique, 15, 285-376.

Zijderveld, J.D.A., Collinson, D., Creer, K.M., Runcorn, S.K., 1967, AC demagnetization of rocks: analysis of results: Methods in paleomagnetism, 1, 254-286. 